\title{
Puberdade precoce
}

\author{
Isabel R. Madeira ${ }^{1, *}$
}

\section{Resumo}

O objetivo do presente artigo é apresentar informações relevantes a respeito de puberdade precoce e de seu diagnóstico diferencial. Foi realizada revisão de literatura científica utilizando busca bibliográfica eletrônica no Medline com as palavras-chave "precocious puberty", "adrenarche" e "gynecomastia" e livros textos contendo dados atualizados relevantes sobre o tema. Foi também consultada a portaria nacional que trata do protocolo clínico e diretrizes terapêuticas em puberdade precoce central. Além da revisão, especialmente no que se refere ao diagnóstico das causas subjacentes e ao diagnóstico diferencial, o artigo apresenta a conduta terapêutica de puberdade precoce verdadeira. A pesquisa de uma causa subjacente está sempre indicada em meninos. Deve ser realizada em meninas com puberdade precoce progressiva, principalmente quando as manifestações se iniciam antes dos seis anos de idade ou naquelas com risco para causas orgânicas, aventadas em função de condições pré-existentes ou sinais e sintomas neurológicos.

Descritores: Puberdade precoce; Adrenarca; Ginecomastia.

\section{Abstract}

\section{Early puberty}

The purpose of this article is to present relevant information about precocious puberty and their differential diagnoses. It was held scientific literature review using electronic literature search in Medline with the keywords "precocious puberty", "adrenarche" and "gynecomastia" and books containing relevant updated data on the subject. It was also consulted the national ordinance that addresses the clinical protocol and therapeutic guidelines for central precocious puberty. The article reviews, especially with regard to diagnosis of the underlying causes and differential diagnosis, and shows the therapeutics options for true precocious puberty. The search for an underlying cause is indicated in progressive precocious puberty in girls, especially those under the age of six years or those with risk to organic causes, suspected in the presence of pre-existing conditions or neurological signs and symptoms. In boys, it should always be performed.

Key words: Precocious puberty; Adrenarche; Gynecomastia.

\section{Resumen}

\section{Pubertad precoz}

El objetivo de este artículo es presentar las informaciones relevantes con respecto a la pubertad precoz y a su diagnóstico
1. Departamento de Pediatria. Faculdade de Ciências Médicas. Universidade do Estado do Rio de Janeiro. Rio de Janeiro, RJ, Brasil.

*Endereço para correspondência:

Rua João da Mata, 128

Rio de Janeiro, RJ, Brasil. CEP 20.511-260.

E-mail: isamadeira@oi.com.br

Revista HUPE, Rio de Janeiro, 2016;15(2):155-162

doi: 10.12957/rhupe.2016.28241

Recebido em 27/06/2016. Aprovado em 04/11/2016.

diferencial. Se revisó la literatura científica y se efectuó una búsqueda bibliográfica electrónica en el Medline con las palaras clave "precocious puberty", "adrenarche" y "gynecomastia" y textos conteniendo datos actualizados relevantes sobre el tema. Se consultó también el decreto nacional que trata sobre el protocolo clínico y las directrices terapéuticas en la pubertad precoz central. Además de la revisión, especialmente en lo que se refiere al diagnóstico de las causas subyacentes y al diagnóstico diferencial, el artículo presenta la conducta terapéutica de la pubertad precoz verdadera. La investigación de una causa subyacente está siempre indicada en niños. Debe ser realizada en niñas con pubertad precoz progresiva, principalmente cuando las manifestaciones se inician antes de los seis años de edad o en aquellas con riesgo por causas orgánicas, presentadas en función de condiciones pre-existentes o señales y síntomas neurológicos.

Palabras clave: Pubertad precoz; Adrenarquía; Ginecomastia.

\section{Introdução}

A puberdade é o período da vida do indivíduo em que, devido à atividade gonadal, surgem as características sexuais secundárias e se adquire a capacidade de reprodução. Neste período ocorre a diminuição da sensibilidade do gonadostato hipotalâmico ao feedback negativo exercido pelos esteroides sexuais, com consequente descarga hipotalâmica do hormônio liberador de gonadotrofinas (GnRH) que estimula a secreção hipofisária dos hormônios luteinizante (LH) e folículo estimulante (FSH). ${ }^{1}$ Estas gonadotrofinas atuam sobre as gônadas, promovendo a gonadarca. Ocorre assim a secreção de esteroides sexuais e a maturação de oócitos e espermatócitos, no sexo feminino e masculino, respectivamente. Os mecanismos responsáveis pela ativação do processo descrito são múltiplos, envol- 


\section{Artigo de revisão}

vendo possivelmente fatores genéticos, metabólicos e outros, incluindo leptina, kisspepetina, neuroquinina B e dinorfina. ${ }^{2-4} \mathrm{O}$ aumento de secreção adrenocortical dos hormônios 17-cetosteroides (dehidroepiandrosterona e sulfato de dehidroepiandrosterona), denominado adrenarca, precede em alguns anos a gonadarca. ${ }^{1}$

Na menina, o evento que marca o início da puberdade é o aparecimento do broto mamário (telarca), e no menino, o aumento dos testículos ( $4 \mathrm{~mL}$ ). A idade de início varia consideravelmente entre os indivíduos, havendo um consenso quanto a faixa de normalidade de 8 a 13 anos para o início da puberdade em meninas, e de 9 a 14 anos em meninos. ${ }^{5,6}$ Nas meninas, o tempo transcorrido entre a telarca e a primeira menstruação (menarca) é de 1,3 a 3,3 anos 7, sendo de duração maior nas que iniciam mais cedo, com consequente preservação do potencial de crescimento. ${ }^{8}$

Durante o processo puberal, inicia-se pilificação na região pubiana (pubarca). A pubarca pode ocorrer a partir de 8 anos no sexo feminino e de 9 anos no masculino, mas geralmente sucede o aparecimento do broto mamário na menina e o aumento do testículo no menino.

Os meninos, além da pubarca, evoluem com aumento do pênis e escurecimento da bolsa escrotal, e $60 \%$ podem apresentar ginecomastia puberal. ${ }^{9}$

A puberdade precoce deve ser considerada sempre que houver aparecimento das características descritas antes de 8 anos na menina ou 9 anos no menino, ou menarca antes de 9,5 anos na menina. ${ }^{5}$

Deve-se, no entanto, levar em conta que, em meninas pertencentes a certas etnias tais como a etnia negra, a presença de telarca ou pubarca a partir dos seis anos de idade pode representar uma variação normal da puberdade. ${ }^{10}$

Um evento normal que deve ser levado em consideração, chamado por alguns autores de minipuberdade, é a presença de mamas nos primeiros meses de vida e de sangramento vaginal nos primeiros dias de vida nas meninas, ou escurecimento do saco escrotal dos meninos nesta idade. É decorrente da ativação do eixo hipotálamo-hipófise-gonadal próprio deste período. ${ }^{11}$

A puberdade precoce pode ser classificada em: completa, ou central, ou gonadotrofina dependente; incompleta, ou periférica, ou gonadotrofina independente. No primeiro caso, a origem é hipotalâmica e o tipo é isossexual, ou seja, as características são compatíveis com o sexo presumido do indivíduo até então. No segundo caso, pode ser do tipo isossexual ou contrassexual, e as causas são: variação normal da pu- berdade; tumor produtor de gonadotrofina coriônica (hCG); hipotireoidismo; síndrome de McCune-Albright; tumor gonadal; hiperplasia congênita de suprarrenal; tumor adrenal; exposição exógena a esteroide sexual; testitoxicose. $^{12}$

O foco deste artigo será a puberdade precoce completa, ou central, ou gonadotrofina dependente, que doravante será denominada puberdade precoce. Os outros tipos serão abordados no diagnóstico diferencial.

\section{Puberdade precoce}

A puberdade precoce completa, ou central, é decorrente de um distúrbio hipotalâmico ou pituitário, sendo gonadotrofina dependente. Pode ser de etiologia neurogênica ou idiopática. Ela é isossexual e pode implicar em maturação completa.

\section{Puberdade precoce em meninas}

A incidência de puberdade precoce é maior em meninas, e nestas, 95\% dos casos são idiopáticos, ocorrendo provavelmente por disparo prematuro do mecanismo normal de puberdade. A alta prevalência neste sexo parece ser decorrente dos níveis mais altos de gonadotrofinas em mulheres. ${ }^{13}$

Os estudos de Chalumeau e colaboradores ${ }^{14}$ e de Cisternino e colaboradores ${ }^{15}$ demonstraram que apenas 8\% das meninas sem achados ao exame neurológico apresentam patologia de sistema nervoso central e que a probabilidade de patologia do sistema nervoso central é inversamente proporcional à idade neste grupo. Nas casuísticas destes autores, apenas 2-7\% das meninas sem achados ao exame neurológico e que iniciaram a puberdade precoce entre 6 e 8 anos apresentam patologia do sistema nervoso central, sendo que apenas 1\% tem tumores como glioma ou astrocitoma.

As meninas com puberdade precoce idiopática têm o desenvolvimento puberal qualitativa e quantitativamente normal. A maioria dos casos é familiar. ${ }^{13}$

A progressão da puberdade pode ser rápida, quando a ativação do eixo hipotálamo-hipófise-ovariano é sustentada. Neste caso, há amadurecimento físico rápido e estirão de crescimento evidente, podendo implicar em deterioração do potencial de crescimento, com consequente baixa estatura na idade adulta. No entanto, o processo em grande parte das vezes não é intenso o suficiente para trazer tal consequência. As meninas que iniciam a puberdade entre 6 e 8 anos de idade e que constituem a maioria dos casos de puberdade precoce, geralmente não se apresentam com curso rapidamente progressivo, e não ocorre dano ao poten- 
cial de crescimento. ${ }^{16}$ Em muitas meninas desta faixa etária, a obesidade parece estar implicada no processo. ${ }^{17}$

A associação entre a obesidade e o desenvolvimento puberal antecipado no sexo feminino poderia ser explicada pelos maiores níveis de leptina na obesidade, já que o hormônio, produzido pelo tecido adiposo, tem papel permissivo sobre a secreção de GnRH em nível hipotalâmico em meninas. ${ }^{2}$

\section{Puberdade precoce em meninos}

Meninos com puberdade precoce devem ser avaliados exaustivamente, uma vez que entre eles este quadro é mais raro do que em meninas, e em razão de muitos apresentarem causas subjacentes. A este respeito, De Sanctis e colaboradores ${ }^{18}$ descreveram em sua casuística a presença de patologia intracraniana em 40\% de meninos com puberdade precoce nos quais não se observavam alterações neurológicas ou neurofibromas ao exame físico.

\section{Etiologia}

Em ambos os sexos, as causas de puberdade precoce central podem ser congênitas, como paralisia cerebral e hidrocefalia, ou adquiridas, como irradiação de sistema nervoso central, traumatismo craniano, inflamação e tumores de sistema nervoso central. ${ }^{19}$

Os tumores podem fazer parte de síndromes específicas, como é o caso do glioma óptico na neurofibromatose do tipo 1, porém mais comumente apresentam-se de forma isolada. As massas tumorais, nesses casos, geralmente correspondem a hamartoma e, mais raramente, a astrocitomas, adenomas, germinomas, pinealomas, ou cistos aracnoides e suprasselares. ${ }^{20}$ Os hamartomas hipotalâmicos são congênitos e constituídos por massa heterotópica de neurônios secretores de GnRH. A puberdade precoce consequente a estes tumores costuma ser diagnosticada no período pré-escolar, podendo estar presentes crises convulsivas do tipo gelásticas. ${ }^{21}$

A puberdade precoce verdadeira também pode ocorrer após o tratamento de uma condição periférica que levou à maturação somática avançada, como por exemplo, a hiperplasia congênita de suprarrenal. A fisiopatologia envolve a retirada do feedback negativo sobre o hipotálamo que vinha sendo exercido pelos elevados níveis de esteroides sexuais inerentes ao distúrbio. ${ }^{22}$

Na casuística de 274 crianças (90,1\% do sexo feminino e $9,9 \%$ do sexo masculino) encaminhadas ao Setor de Endocrinologia Pediátrica do Hospital Universitário Pedro Ernesto da Universidade do Estado do Rio de Janeiro (HUPE-UERJ) para investigação de precocidade sexual, a puberdade precoce verdadeira foi diagnosticada em 83 crianças (30,3\% dos casos). Neste grupo, $73(87,9 \%)$ eram meninas e 10 (12,1\%) eram meninos. O acometimento do sistema nervoso central foi documentado em 19 crianças $(22,2 \%)$ dos casos. A prevalência de acometimento do sistema nervoso central excede a relatada na literatura e pode ser explicada pelo encaminhamento de pacientes mais graves para um hospital de referência com serviços especializados em endocrinologia, neurologia e neurocirurgia. ${ }^{23}$

\section{Diagnóstico}

Na anamnese deve-se valorizar a época de aparecimento e evolução das características puberais, o uso de medicamentos tópicos ou sistêmicos, não só pela criança, mas pela mãe ou cuidador. Como exemplo, temos o caso de criança cuja mãe esteja utilizando estrogênio transdérmico, ou amamentada ao seio de mãe em uso de contraceptivo com estrogênio. É importante também a história gestacional, parto e nascimento, pois é sabido, por exemplo, que crianças nascidas pequenas para a idade gestacional podem entrar em puberdade mais cedo. ${ }^{24} \mathrm{Na}$ história familiar, deve-se pesquisar a estatura dos pais e sua época de puberdade. Deve ser realizado exame físico completo, sendo mandatória a avaliação das características sexuais puberais. A classificação de Tanner $^{25}$ para os estágios puberais é sempre empregada. A avaliação da pele com a criança despida não pode ser esquecida, buscando-se especialmente manchas café com leite, típicas da síndrome de McCune-Albright ou as lesões típicas da neurofibromatose do tipo 1. O exame neurológico pode direcionar para causas neurogênicas da puberdade precoce. No caso de sangramento vaginal isolado, avaliar a possibilidade de vulvovaginite, corpo estranho, abuso sexual, ou tumor do trato genital. A história e o exame físico vão direcionar para os exames complementares a serem solicitados.

\section{Exames de imagem}

A radiografia de punho e mão esquerdos para a informação quanto à idade óssea é sempre solicitada nos casos de puberdade precoce. ${ }^{26} \mathrm{Em}$ meninas, quando a anamnese e o exame físico sugerem o diagnóstico de pubarca ou telarca precoce, a radiografia de punho e mão esquerdos pode ser o único exame complementar indicado em uma primeira avaliação. Se a idade óssea não está anormalmente avançada em relação à idade cronológica e à estatural, possivelmente trata-se de variação da normalidade e deve-se realizar apenas o acompanhamento clínico. ${ }^{27}$ 


\section{Artigo de revisão}

A radiografia de punho e mão é também muito importante para a avaliação de meninos e meninas com puberdade precoce neurogênica, especialmente em crianças com história de irradiação craniana, nas quais pode haver concomitante deficiência de hormônio do crescimento. Nestes casos, a idade óssea estará mais avançada que a idade estatural. ${ }^{28}$

A idade óssea serve ainda para o cálculo de previsão da estatura final. Quando existe prognóstico de baixa estatura na idade adulta, há a indicação do bloqueio da puberdade precoce. ${ }^{13}$

A ultrassonografia da pelve é útil para determinar o tamanho e a morfologia uterina, além da presença de cistos ovarianos. Comprimento uterino maior que 3,4 a 4 cm, ${ }^{29}$ volume maior que $2 \mathrm{cc}$, relação corpo/colo maior que $1^{20}$ e espessura endometrial acima de $2 \mathrm{~mm}$ são indicativos de estrogenização. ${ }^{30} \mathrm{O}$ ponto de corte para volume ovariano puberal varia de 1 a 3 cc. ${ }^{30}$ A presença de um cisto ovariano de $10 \mathrm{~mm}$ de diâmetro ou mais geralmente representa um folículo pré-ovulatório transitório, mas a possibilidade de um cisto persistente deve ser avaliada com repetição do exame. ${ }^{31}$

A ultrassonografia dos testículos deve ser realizada nos casos em que há aumento testicular unilateral em busca de tumor. ${ }^{12}$

A ultrassonografia do abdome com ênfase nas suprarrenais, avaliada por um ultrassonografista experiente, tem boa sensibilidade no diagnóstico de tumores de suprarrenal. ${ }^{32}$

Na dependência da clínica e dos exames anteriores, pode haver a indicação de se realizar tomografia computadorizada ou ressonância magnética para melhor visualização de tumores adrenais ou gonadais. ${ }^{32}$

A ressonância magnética da região hipotálamo-hipofisária deve ser sempre realizada para a investigação etiológica da puberdade precoce em meninos e está indicada em meninas na puberdade precoce progressiva, especialmente naquelas com menos de seis anos de idade ou naquelas com risco para causas orgânicas, que devem ser aventadas em função de condições pré-existentes ou sinais e sintomas neurológicos. ${ }^{33}$

\section{Avaliação hormonal}

Os principais hormônios a serem solicitados são LH, FSH, estradiol, testosterona, sulfato de dehidroepiandrosterona (DHEAS), 17-hidroxiprogesterona (17OHP), hormônio estimulador da tireoide (TSH), tiroxinalivre (T4 livre) e prolactina (PRL). Outros exames a serem considerados são a dosagem de hCG e os testes de estímulo com GnRH com dosagem de gonadotrofinas e com hormônio adrenocorticotrófico (ACTH) com dosagem de $17 \mathrm{OHP}$. $^{1,12,13}$

Na puberdade precoce esperam-se níveis puberais de LH, FSH e estradiol em meninas e de LH, FSH e testosterona em meninos. Os valores de LH por ensaio imunoquiminulométrico são os mais informativos. Dosagens abaixo de 0,1 IU/L afastam puberdade precoce. Resende e colaboradores ${ }^{34}$ demonstraram que o ponto de corte de 0,2 IU/L distingue meninos normais pré-púberes de púberes com 100\% de sensibilidade e especificidade, mas que 50\% das meninas com broto mamário têm níveis abaixo deste ponto de corte.

No início do quadro, pode ser necessário o teste de estímulo com GnRH para se comprovar a puberdade precoce verdadeira. ${ }^{13}$ A dosagem de LH após estímulo com GnRH mostra sobreposição de valores entre crianças impúberes e aquelas na fase inicial da puberdade. Os pontos de corte não estão bem estabelecidos, mas a literatura sugere valores entre 3,3 e $5 \mathrm{IU} / \mathrm{L}^{.}{ }^{34,35}$

A avaliação dos outros hormônios mencionados pode ser também importante na investigação etiológica da puberdade precoce. O DHEAS é um marcador de carcinoma de suprarrenal e a $17 \mathrm{OHP}$ diagnostica a hiperplasia congênita de suprarrenal por deficiência de 21-hidroxilase. ${ }^{36}$ A avaliação da função tireoidiana é pertinente porque o hipotireoidismo prolongado pode levar a telarca e até menarca em meninas ${ }^{37}$ e a macroorquidismo em meninos. ${ }^{12}$ A dosagem de PRL está indicada porque os prolactinomas podem provocar telarca em meninas ${ }^{1}$ e ginecomastia em meninos, ${ }^{12}$ e a dosagem de hCG visa a afastar o diagnóstico de tumores produtores deste hormônio, principalmente em meninos. ${ }^{12}$

O esfregaço da mucosa vaginal pode ser útil em indicar estrogenização quando há mudança do padrão pré-púbere, com predominância das células basais, para o padrão estrogenizado, com predominância das células superficiais. ${ }^{21}$

\section{Diagnóstico diferencial}

$\mathrm{Na}$ avaliação etiológica da precocidade sexual é necessário afastar o uso de medicamentos tópicos ou sistêmicos pela criança ou pelo cuidador, além de outras condições, incluindo a telarca e a pubarca prematuras, a hiperplasia congênita de suprarrenal, os tumores gonadais e de suprarrenais, a síndrome de McCune-Albright, a testitoxicose e o hipotireoidismo. Todas estas são formas de puberdade incompleta, ou periférica, ou gonadotrofina independente. 


\section{Telarca prematura}

Neste quadro, próprio do sexo feminino, ocorre desenvolvimento mamário isolado, não havendo outros caracteres sexuais secundários nem aceleração da velocidade de crescimento ou da idade óssea. A fisiopatologia parece estar relacionada a aumento da sensibilidade mamária ao estrogênio, ou secreção transitória de estrogênio por cistos foliculares ovarianos, ou aumento da produção de estrogênios a partir de precursores adrenais, ou ainda ativação transitória parcial do eixo hipotálamo-hipófise-ovariano, com secreção excessiva de FSH. A possibilidade de aumento de estrogênio na dieta contemporânea também é aventada. No teste de estímulo com GnRH há resposta predominantemente do FSH, embora este teste não seja necessário, por se tratar de condição benigna. Sendo assim, a conduta recomendada é o acompanhamento clínico, já que a telarca pode ser o primeiro sinal da puberdade precoce verdadeira. ${ }^{27}$

\section{Pubarca prematura}

A pubarca prematura é a causa mais comum de pubarca precoce isolada. Vem acompanhada de odor axilar e por vezes de acne discretíssima, sem outros sinais de maturação sexual. Não há virilização, a menina não apresenta clitoriomegalia, e o menino não desenvolve aumento do pênis. Não há aceleração significante da velocidade de crescimento nem da maturação esquelética. Sua fisiopatologia envolve a adrenarca prematura ou exagerada. ${ }^{27}$ Observa-se aumento modesto dos níveis de sulfato de dehidroepiandrosterona. Trata-se de um diagnóstico de exclusão, depois de afastada a possibilidade de tumores de suprarrenais e gonadais e hiperplasia congênita de suprarrenais, cujo diagnóstico está descrito a seguir.

\section{Hiperplasia congênita de suprarrenal}

A hiperplasia congênita de suprarrenal decorre, em 95\% dos casos, da deficiência da enzima 21-hidroxilase. As meninas com as formas clássicas (perdedora de sal ou virilizante simples) já nascem com genitália ambígua enquanto os meninos nascem com genitália normal e após o segundo ano de vida, aceleram o crescimento e apresentam não só pubarca, como aumento do pênis e hipertrofia muscular, mas sem aumento dos testículos. Há aceleração da maturação esquelética e se o tratamento é tardio ou inadequado, pode ocorrer puberdade precoce verdadeira. A forma não clássica ou tardia causa pubarca precoce em meninas e meninos, que também não apresentam aumento dos testículos. Pode haver aceleração de crescimento e de maturação esquelética. ${ }^{36}$

O diagnóstico é feito por meio da dosagem de 17OHP. Segundo o consenso da Endocrine Society, ${ }^{36}$ se esta dosagem for menor que $200 \mathrm{ng} / \mathrm{dL}$, o diagnóstico é improvável. Por outro lado, se for maior que 10.000 ng/dL, é provável o diagnóstico da forma clássica. Se a dosagem for entre 200 e $10.000 \mathrm{ng} / \mathrm{dL}$, deve ser realizado o teste de estímulo com ACTH. Após o teste, níveis acima de $10.000 \mathrm{ng} / \mathrm{dL}$ confirmam o diagnóstico da forma clássica. Níveis entre 1.000 e 10.000 ng/dL são compatíveis com a forma não clássica e níveis menores que $990 \mathrm{ng} / \mathrm{dL}$ tornam os diagnósticos pouco prováveis.

\section{Tumores adrenais}

No carcinoma de suprarrenal ocorre virilização e aumento importante deDHEAS, e os exames de imagem confirmam o diagnóstico. ${ }^{32}$

\section{Tumores gonadais}

Os tumores gonadais podem causar puberdade, desenvolvimento puberal isosexual ou contrassexual. Nos tumores testiculares, observa-se aumento testicular unilateral ao exame físico. ${ }^{12} \mathrm{Em}$ todos os tumores gonadais, os exames de imagem são importantes para o diagnóstico. ${ }^{31}$

\section{Tumor produtor de hCG}

São raros em crianças e podem ser hepatoblastomas, pinealomas ou tumores retroperitoneais. Manifestam-se clinicamente com virilização em meninos, sendo o diagnóstico histológico, após investigação por imagem.12

\section{Síndrome de McCune-Albright}

A síndrome cursa com manchas café com leite, displasia fibrosa poliostótica no crânio e nos ossos longos e puberdade precoce periférica, esta mais comumente observada em meninas. A puberdade precoce é consequência da hiperfunção autônoma dos ovários, e esta hiperfunção também pode estar presente em outras glândulas. Em prevalência decrescente, podem coexistir hipertireoidismo, hipercortisolismo adrenal, aumento de hormônio de crescimento e de prolactina. Cistos ovarianos grandes e unilaterais podem ser visualizados à ultrassonografia. Os níveis de gonadotrofinas estarão baixos. ${ }^{38}$

\section{Testitoxicose}

Trata-se de desordem familial, autossômica dominante, mas com penetração variável, com hiperplasia 


\section{Artigo de revisão}

de células de Leydig e maturação das células germinativas. Ao exame físico, observa-se aumento testicular bilateral. Laboratorialmente, os níveis de gonadotrofinas estarão baixos. ${ }^{39}$

\section{Hipotireoidismo}

O hipotireoidismo prolongado, sem tratamento, pode provocar telarca com galactorreia e até menarca, ${ }^{37}$ além de macroorquidismo em meninos. ${ }^{12}$ Geralmente não ocorre pubarca. É importante ressaltar que esta é a única forma de precocidade sexual em que há desaceleração do crescimento. A maturação esquelética também está atrasada e à ultrassonografia, podem-se observar ovários multicísticos em meninas. Os níveis de LH estão baixos e os de FSH próximos daqueles encontrados no início da puberdade. O TSH e a PRL estão elevados. ${ }^{37}$

$\mathrm{Na}$ casuística de 274 crianças acompanhadas por precocidade sexual no Setor de Endocrinologia Pediátrica do HUPE-UERJ, 109 (39,8\%) tiveram como diagnóstico pubarca prematura, 83 (30,3\%) puberdade precoce verdadeira, 59 (21,5\%) telarca prematura e 23 $(8,4 \%)$ outras causas de puberdade precoce periférica. Este último grupo foi composto por $9(39,1 \%)$ crianças com puberdade precoce isosexual (6 com hiperplasia congênita de suprarrenal e 3 com síndrome de McCune-Albright) e 14 (60,9\%) contrassexual (13 com hiperplasia congênita de suprarrenal e 1 com tumor de suprarrenal). ${ }^{23}$

\section{Tratamento}

O tratamento da puberdade precoce verdadeira se faz por meio do bloqueio desta com análogos do GnRH(GnRHa). Este procedimento tem como objetivos principais a preservação do potencial de crescimento e a prevenção de desajuste entre a maturação física e a psicossocial nestas crianças. Existem critérios clínicos e laboratoriais para tal tratamento. O critério clínico mais importante é a progressão da puberdade, já que muitos pacientes com puberdade precoce têm apresentação de evolução lenta ou mesmo não progressiva, e atingem estatura adulta compatível com os padrões familiares. Outros elementos a serem considerados são a velocidade de crescimento e a maturação esquelética. ${ }^{13}$

O critério laboratorial tem como pilares a dosagem do LH basal e estimulado, bem como dos esteroides sexuais. As sociedades internacionais de endocrinologia pediátrica publicaram, em 2009, um consenso de seus especialistas ${ }^{13}$ que afirma que as meninas com puberdade precoce progressiva iniciada antes dos 6 anos de idade são as que certamente se beneficiariam, com o tratamento, em termos de preservação de estatura final. Acrescentam ainda que a decisão de iniciar tratamento em meninas que iniciaram a puberdade após os 6 anos deve ser individualizada. Consta do documento que o tratamento deve ser considerado para todos os meninos com puberdade precoce progressiva iniciada antes dos 9 anos de idade que apresentem comprometimento no potencial de crescimento. O grupo afirma que o emprego do GnRHa somente com o intuito de influenciar aspectos psicossociais deve ser analisado cuidadosamente, já que não existem dados convincentes na literatura que o suportem.

O Ministério da Saúde Brasileiro (MS ${ }^{40}$ preconiza o tratamento para crianças com 8 anos ou menos que preencham os critérios acima estabelecidos. Contraindica o tratamento para meninas com idade óssea acima de 12 anos e para meninos com idade óssea acima de 13 anos.

A dose preconizada pelo MS é de $3,75 \mathrm{mg}$ a cada mês, via intramuscular, de leuprorrelina ou de triptorrelina. Alternativamente, a medicação pode ser empregada na dose de 11,25 mg a cada três meses. ${ }^{41}$ Segundo o MS, o tratamento deve ser realizado até a idade cronológica normal para o desenvolvimento da puberdade, com idade óssea entre 12 e 12,5 anos na menina e entre 13 e 13,5 anos no menino.

A associação do hormônio de crescimento ao tratamento com GnRHa, com o objetivo de otimizar a estatura final, tem sido empregada, ${ }^{41}$ mas ainda não é consensual. ${ }^{13}$

O tratamento cirúrgico dos tumores de sistema nervoso central, quando estes são a causa da puberdade precoce, deve ser particularizado. A ressecção cirúrgica dos hamartomas hipotalâmicos raramente é realizada, e o seguimento deve ser conjunto com o neurocirurgião. ${ }^{40} \mathrm{O}$ tratamento das causas de puberdade precoce periférica é específico para cada uma das etiologias e foge ao escopo deste artigo.

\section{Conclusão}

O conhecimento da puberdade normal e de suas variantes é essencial para o diagnóstico da puberdade precoce e para a indicação oportuna de seu tratamento. A pesquisa de uma causa subjacente está indicada na puberdade precoce progressiva em meninas, em especial naquelas com menos de 6 anos de idade ou naquelas com risco para causas orgânicas, que devem ser aventadas em função de condições pré-existentes ou sinais e sintomas neurológicos. Em meninos, a pesquisa deve sempre ser realizada. 


\section{Referências}

1. Rosenfield RL, Cooke DW, Radovick S. Puberty and its disorders in the female. In: Sperling MA (ed.). Pediatric Endocrinology. 4th ed. Philadelphia: Elsevier, Sauders;2014.p.569-663.

2. Sabchez-Garrido MA, Tena-Sempere M. Metabolic control of puberty: roles of leptina and kisspeptins. Horm Behav. 2013;64:187-94. http://doi.org/10.1016/j.yhbeh.2013.01.014

3. Abreu AP, Kaiser UB. Pubertal development and regulation. Lancet Diabetes Endocrinol. 2016;4:254-64. http://doi. org/10.1016/S2213-8587(15)00418-0

4. Livadas S, Chrousos GP. Control of the onset of puberty. Curr Opin Pediatr. 2016;28:551-8. http://doi.org/ 10.1097/ MOP.0000000000000386

5. Rosenfiel RL, Bordini B, Yu C. Comparison of detection of normal puberty in girls by a hormonal sleep test and a gonadotropin-releasing hormone agonist test. J Clin Endocrinol Metab. 2013;98:1591-601. http://doi.org/10.1210/jc.2012-4136

6. Halan WR, Grillo GP, Cornoni-Huntley J, et al. Secondary sex characteristics of boys 12 to 17 years of age: the U.S. Health Examination Survey. J Pediatr. 1979;95:293-7.

7. Marshall W, Tanner J. Variations in pattern of pubertal changes in girls. Arch Di Child. 1969;44:291-8.

8. Mart-Henneberg C, Vizmanos B. The duration of puberty in girls is related to the timing of its onset. $\mathrm{J}$ Pediatr. 1997;131:618-21.

9. Glass AR. Gynecomastia. Endocrinol Metab Clin North Am. 1994;23:825-37.

10. Parent AS, Teilmann G, Juul A, et al. The timing of normal puberty and the age limits of sexual precocity: variations around the world, secular trends, and changes after migration. Endocr Rev. 2003;24:668-93. http://doi.org/10.1210/er.2002-0019

11. Winter JSD, Hughes IA, Reyes FL, et al. Pituitary-gonadal relations in infancy: patterns of serum gonadal steroid concentrations in man from birth to two years of age. J Clin Endocrinol Metab 1976;42:679-86. http://doi.org/10.1210/jcem-42-4-679

12. Palmert MR, Dunkel L, Witchel SF. Puberty and its disorders in the male. In: Sperling MA (ed.). Pediatric Endocrinology. 4th ed. Philadelphia: Elsevier, Sauders; 2014. p.697-733.

13. Carel JC, Eugster EA, Rogol A, et al. Consensus statement on the use of gonadotropin-releasing hormone analogs in children. Pediatrics. 2009;123:e752-62. http://doi.org/10.1542/ peds.2008-1783

14. Chalumeau M, Hadjiathanasiou CG, Ng SM, et al. Selecting girls with precocious puberty for brain imaging: validation of European evidence-based diagnosis rule. J Pediatr. 2003:143:445-50. http://doi.org/10.1067/S00223476(03)00328-7

15. Cisternino M, Arrigo T, Pasquino AM, et al. Etiology and age incidence of precocious puberty in girls: a multicentric study. J Pediatr Endocrinol Metab. 2000;13:695-701.

16. Brauner R, Adan L, Malandry E, et al. Adult hight in girls with idioptic true precocious puberty. J Clin Endocrinol Metab. 1994;79:415-20. http://doi.org/ 10.1210/jcem.79.2.8045957

17. Rosenfield RL, Lipoton RB, Drum ML. Thelarche, pubarche, and menarche attainment in children with normal and elevated body mass index. Pediatrics. 2009;123:84-8. http://doi. org/10.1542/peds.2008-0146

18. De Sanctis V, Corrias A, Rizzo V, et al. Etiology of central precocious puberty in males: the results of the Italian Study Group for Physiopathology of Puberty. J Pediatr Endocrinol Metab. 2000;13:687-93.
19. Junier MP, Wolff A, Hoffman G, et al. Effect of hypothalamic lesions that induce precocious puberty on the morphological and functional maturation of the luteinizing hormone-releasing hormone neuronal system. Endocrinology. 1992;131:787-98. http://doi.org/10.1210/endo.131.2.1639024

20. Carel JC, Leger J. Clinical practice: precocious puberty. N Engl J Med. 2008;358:2366-77. http://doi.org/10.1056/NEJMcp0800459

21. Nathan BN, Palmer MR. Regulation and disorders of pubertal timing. Endocrinol Metab Clin North Am. 2005;34:617-41. http://doi.org/10.1016/j.ecl.2005.04.015

22. Pescovitz OH, Comite E, Cassorla E, et al. True precocious puberty complicating congenital adrenal hyperplasia: treatment with a luteinizing hormone-releasing hormone analog. J Clin Endocrinol Metab. 1984;58:857-64. http://doi.org/10.1210/ jcem-58-5-857

23. Gazolla FM, Rodrigues GC, Alencar RC, et al. Perfil de uma amostra de precocidade sexual acompanhada em um ambulatório de endocrinologia pediátrica. Arq Bras Endocrinol Metab. 2007;51:S757-S64.

24. Clayton PE, Cianfarani S, Czernichow P, et al. Management of the child born small for gesational age through to adulthood: a consensus statement of the International Societies of Pediatric Endocrinology and the Growth Hormone Research Society. J Clin Endocrinol Metab. 2007;92:804-10. http://doi.org/10.1210/ jc.2006-2017

25. Marchall WA, Tanner JM. Variations in the pattern of pubertal changes in girls and boys. Arch Dis Child. 1969;44:291-303.

26. Greulich WW, Pyle SI. Radiographic atlas of skeletal development of the hand and wrist. 2nd ed. Stanford: Stanford University Press; 1959. 255p.

27. Rosenfield RL Normal and almost normal variants of precocious puberty: premature pubarche and premature thelarche revisited. Horm Res. 1994;41:7-13.

28. Kraiter M, Cara J, Rosenfield R. Modifying the outcome of complete precocious puberty: to treat or not to treat. In Grave G, Cutler G (ed). Sexula Precocity: Etiology, Diagnosis, and Management. New York: Haven Press;1993. p. 109-120.

29. de Vries L, Horev G, Schwrtz M, et al. Ultrassonographic and clinical parameters for early differentiation between precocious puberty and premature thelarche. Eu J Endocrinol. 2006:154:891-8. http://doi.org/10.1530/eje.1.02151

30. Haber HP, Wollmann HA, Ranke MB. Pelvic ultrasonography: early differentiation between isolated premature thelarche and central precocious puberty. Eur J Pediatr. 1995;154:182-6.

31. Salardi S, Orsini L, Cacciari E, et al. Pelvic ultrassonography in girls with precocious puberty, congenital adrenal hyperplasia, obesity, or hirsutism. J Pediatr 1988;112:880-7.

32. Balassi C, Navarro OM, Daneman A. Adrenal masses in children. Radiol Clin North Am. 2011;49:711-27. http://doi. org/10.1016/j.rcl.2011.05.001

33. Grunt JA, Midyett LK, Simon SD, el al. When should cranial magnetic resonance imaging be used in girls with early sexual development? J Pediatr Endocrinol Metab. 2004;17:775-80.

34. Resende EA, Lara BH, Reis JD, et al. Assessment of basal and gonadotropin-releasing hormone-stimulated gonadotropins by immunochemiluminometric and immunofluorometric assays in normal children. J Clin Endocrinol Metab. 2007;92:1424-9. http://doi.org/10.1210/jc.2006-1569

35. Neely EK, Wilson DM, Lee PA, et al. Spontaneous serum gonadotropin concentrations in the evaluation of precocious puberty. J Pediatr. 1995;127:47-52. 


\section{Artigo de revisão}

36. Speiser PW, Azziz R, Baskin LS, et al. Congenital adrenal hyperplasia due to steroid 21-hydroxylase deficiency: an Endocrine Society clinical practice guideline. J Clin Endocrinol Metab. 2010;95:4133-60. http://doi.org/10.1210/jc.2009-2631

37. Van Wyk J, Grumbach M. Syndrome of precocious menstruation and galactorrhea in juvenile hypothyroidism: an example of hormonal overlap in pituitary feedback. J Pediatr. 1960;57:416-23.

38. VölkI TMK, Dörr HG. McCune-Albright syndrome: clinical picture and a natural history in children and adolescents. J Pediatr Endocrinol Metab. 2006;19:551-9.

39. Shenker A, Laue L, Kosugi S, et al. A constitutively activating mutation of the luteinizing hormone receptor in familial male precocious puberty. Nature. 1993;365:652-54. http://doi. org/10.1038/365652a0

40. Brasil. Ministério da Saúde. Secretaria de Atenção à Saúde.
Portaria № 111, de 23 de abril de 2010. Protocolo Clínico e Diretrizes Terapêuticas. Puberdade Precoce Central.

http://bvsms.saude.gov.br/bvs/saudelegis/sas/2010/ prt0111_23_04_2010_rep.html. Acesso em: 22/3/2016.

41. Brito VN, Spinola-Castro AM, Kochi C, et al. Central precocious puberty: revising the diagnosis and therapeutic management. Arch Endocrinol Metab. 2016;60:163-72. http://doi. org/10.1590/2359-3997000000144

42. Liu S, Liu Q, Cheng X, et al. Effects and safety of combination therapy with gonadotropin-releasing hormone analogue and growth hormone in girls with idiopathic central precocious puberty: a meta-analysis. J Endocrinol Invest. 2016. [Epub ahead of print]. http://www.ncbi.nlm.nih.gov/pubmed/27225286. Acesso: 04/9/2016 